\title{
Nonmuscle myosin heavy chain IIA mediates integrin LFA-1 de-adhesion during $\mathrm{T}$ lymphocyte migration
}

\author{
Nicole A. Morin, ${ }^{1}$ Patrick W. Oakes, ${ }^{2}$ Young-Min Hyun, ${ }^{6}$ Dooyoung Lee, ${ }^{4}$ \\ Y. Eugene Chin, ${ }^{1}$ Michael R. King, ${ }^{5}$ Timothy A. Springer, ${ }^{3}$ \\ Motomu Shimaoka, ${ }^{3}$ Jay X. Tang, ${ }^{2}$ Jonathan S. Reichner, ${ }^{1}$ \\ and Minsoo $\mathrm{Kim}^{6}$
}

\begin{abstract}
'Department of Surgery, Rhode Island Hospital and Brown Medical School, Providence, RI 02903
2Department of Physics, Brown University, Providence, RI 02912

${ }^{3}$ The CBR Institute for Biomedical Research, Harvard Medical School, Boston, MA 02115

${ }^{4}$ Department of Chemical Engineering, ${ }^{5}$ Department of Biomedical Engineering, and ${ }^{6}$ Department of Microbiology and Immunology, David H. Smith Center for Vaccine Biology and Immunology, Aab Institute of Biomedical Sciences, University of Rochester, Rochester, NY 14642
\end{abstract}

Precise spatial and temporal regulation of cell adhesion and de-adhesion is critical for dynamic lymphocyte migration. Although a great deal of information has been learned about integrin lymphocyte function-associated antigen (LFA)-1 adhesion, the mechanism that regulates efficient LFA-1 de-adhesion from intercellular adhesion molecule (ICAM)-1 during $T$ lymphocyte migration is unknown. Here, we show that nonmuscle myosin heavy chain IIA (MyH9) is recruited to LFA-1 at the uropod of migrating T lymphocytes, and inhibition of the association of MyH9 with LFA-1 results in extreme uropod elongation, defective tail detachment, and decreased lymphocyte migration on ICAM-1, without affecting LFA-1 activation by chemokine CXCL-12. This defect was reversed by a small molecule antagonist that inhibits both LFA-1 affinity and avidity regulation, but not by an antagonist that inhibits only affinity regulation. Total internal reflection fluorescence microscopy of the contact zone between migrating T lymphocytes and ICAM-1 substrate revealed that inactive LFA-1 is selectively localized to the posterior of polarized T lymphocytes, whereas active LFA- 1 is localized to their anterior. Thus, during T lymphocyte migration, uropodal adhesion depends on LFA-1 avidity, where MyH9 serves as a key mechanical link between LFA-1 and the cytoskeleton that is critical for LFA-1 de-adhesion.

\section{CORRESPONDENCE}

Minsoo Kim:

minsoo_kim@urmc.rochester.edu

Abbreviations used: DIC, differential interference contrast; ICAM, intercellular adhesion molecule; MLC, myosin regulatory light chain; MyH9, nonmuscle myosin heavy chain IIA; ROCK, Rho-associated kinase; siRNA, small interfering RNA; TIRF, total internal reflection fluorescence.
By contributing to the positioning of immunocompetent cells throughout the body, leukocyte migration plays a central role in the function of the immune system. Extravasation of blood leukocytes is critical for immune surveillance and is a crucial step in the development of inflammatory responses. During migration, integrins mediate leukocyte adhesion to the extracellular matrix or other cells and function as traction sites over which leukocytes migrate. Integrins are a family of $\alpha \beta$ heterodimeric membrane receptors that mediate cell-cell, cell-extracellular matrix, and cell-pathogen interactions. The integrin LFA-1 (or $\left.\alpha_{L} \beta_{2}\right)$ is expressed on most leukocytes and plays a major role in regulating leukocyte adhesion and recruitment to damaged or infected tissues during inflammation.
Extracellular signals such as chemokines stimulate $G$ protein-coupled receptors and their associated signaling cascades ("inside-out" signals), resulting in dramatic changes in cell shape and eventually leukocyte polarization. Polarized leukocytes exhibit dynamic spatio-temporal regulation of intracellular signaling events and cytoskeletal rearrangement. Precise spatial and temporal regulation of integrin activation occurs during the directional migration of these cells. The process of leukocyte migration can be conceptualized as a cyclic process that involves at least three interdependent events, including de novo cell adhesion at the leading edge, cell contraction at the cell body, and detachment at the trailing edge. Active integrins mediate adhesion at the leading edge to provide traction, and de-adhesion by inactive integrins takes place at 
the trailing edge to allow for leukocyte detachment and forward movement (1). Therefore, both integrin-mediated cell adhesion and de-adhesion are essential for efficient cell migration.

Several components of the inside-out signaling pathways involved in the regulation of LFA-1 adhesiveness have been identified. After being freed from the rod domain by proteolysis or truncation, talin head domain activates integrins by directly binding to $\beta$ subunits $(2-5)$. Mutant talin resistant to calpain proteolysis reduces adhesion turnover during cell migration (6). Rap-1, a member of Ras family of small GTPases, and its interacting molecule, RAPL, mediate TCR- and chemokine-induced adhesion of leukocytes to intercellular adhesion molecule (ICAM)-1 and vascular cell adhesion molecule $1(7,8)$. Although an extensive amount of information has been gathered from the intensive investigation of integrins, most studies have focused on the signaling processes associated with integrin activation and cell adhesion. However, the mechanisms for integrin inactivation or deadhesion during cell migration are equally important and remain uncharacterized.

LFA-1 ligand binding activity is controlled by two distinct mechanisms, "affinity" regulation and "avidity" (or "valency") regulation. In the former, conformational changes in the protein impart high affinity ligand binding. In the latter, membrane redistribution of LFA-1 functions to strengthen overall cellular adhesiveness. During inside-out signaling, stimuli received by a variety of cell surface receptors initiate intracellular signaling that impinges upon integrin cytoplasmic domains and disassociates the cytoplasmic tails of $\alpha_{L}$ and $\beta_{2}$ subunits (2, 9-11). These events lead to disruption of the association between the integrin head and legs $(10,12)$. The resultant switchblade-like opening of the integrin molecule is linked to the active high affinity conformation that binds extrinsic ligands such as ICAMs (13-15). In addition to alterations in the conformation of individual integrin $\alpha \beta$ heterodimers, the overall strength of cellular adhesive interactions is also regulated by the total number of bonds formed (16). The number of adhesive bonds that can form is mainly mediated by changes in the cell surface receptor diffusivity or local clustering, and ligand binding-dependent redistribution as a consequence of adhesion strengthening (8, 13, 16-21). Although LFA-1 affinity and avidity is thought to be tightly and dynamically regulated during $\mathrm{T}$ lymphocyte migration, the relative importance of the different states of integrin activation during cell migration remains unknown. Recently, we have assessed the effects of two distinct classes of small molecule allosteric LFA-1 antagonists on LFA-1 regulation (16). The $\alpha$ I allosteric antagonist, BIRT377, binds to the $\alpha_{\mathrm{L}}$ I domain and stabilizes it in the low affinity closed conformation. In contrast, the $\alpha / \beta$ I-like allosteric antagonist, XVA143, binds to the metal-ion-dependent adhesion site of the $\beta_{2}$ I-like domain and also, in part, to the $\alpha_{\mathrm{L}}$ subunit. XVA143 blocks the communication of conformational signals from the $\beta_{2}$ I-like domain to the $\alpha_{\mathrm{L}}$ I domain; therefore, it blocks inside-out affinity enhancement (16). BIRT377 inhibits both affinity and avidity regulation of LFA-1 by stabilizing the I domain in the low affinity state. Whereas, XVA143 inhibits only affinity regulation activation, suggesting that avidity regulation does not require conformational change in response to signals from within the cell, but still requires conversion of the $\alpha \mathrm{L}$ I domain to the high affinity and open conformation driven by ICAM-1 binding (16). Therefore, these reagents are excellent tools for distinguishing between affinity and avidity regulation of LFA-1 adhesiveness during cell migration.

Myosins are actin-dependent molecular motors that use the energy of ATP hydrolysis to move along actin filaments. The myosin family consists of at least 20 structurally and functionally distinct classes (22). Myosin heavy chains typically consist of three distinct regions, including an N-terminal motor or head domain responsible for actin binding and ATP hydrolysis, a neck region that binds light chains or calmodulin, and a C-terminal tail that is responsible for cargo binding and/ or dimerization of heavy chains. The myosin II family can be divided into nonmuscle cytoplasmic, cardiac muscle, smooth muscle, and skeletal muscle subclasses, each with multiple isoforms (23). Myosin II in nonmuscle cells plays important roles in cytokinesis and cell migration (24). In Dictyostelium, myosin II gene knockout reduces migration speed and results in a loss of forward bias in protrusion (25). Genomic analysis has identified at least three different nonmuscle myosin heavy chain type II isoforms in humans termed nonmuscle myosin heavy chain IIA (MyH9), nonmuscle myosin heavy chain IIB (MyH10), and nonmuscle myosin heavy chain IIC (MyH14) (23). Most cell types contain both MyH9 and MyH10. However, some are selectively enriched either in $\mathrm{MyH} 9$ (e.g., platelets, lymphocytes, neutrophil granulocytes, brush border cells, and RBL2H3 cells) (26) or in MyH10 (e.g., neurons) (27). Here, we provide evidence that adhesion of the tail of migrating $\mathrm{T}$ lymphocytes is mediated by avidity-regulated LFA-1 and that MyH9 plays a key role in LFA-1 de-adhesion and tail retraction.

\section{RESULTS}

ICAM-1 binding recruits MyH9 to LFA-1

To identify key cytoplasmic molecules mediating LFA-1-dependent T lymphocyte migration on ICAM-1, LFA-1 immunoprecipitates obtained from human primary $\mathrm{T}$ lymphocytes adherent to four different substrate conditions were analyzed (Fig. 1). First, $\mathrm{T}$ lymphocytes were exposed to $\mathrm{Mg}^{2+}$ /EGTA and, thus, cell surface LFA-1 was constitutively active. Both on poly-L lysine and on ICAM-1 surfaces, T lymphocytes spread out, but no polarization or migration was observed (Fig. 1 A and Videos S1 and S2, which are available at http://www.jem .org/cgi/content/full/jem.20071543/DC1). This suggests that LFA-1/ICAM-1 binding ("outside-in" signal) is not sufficient to induce cell polarization and migration. Next, when T lymphocytes were placed on a poly-L lysine/CXCL-12 surface in buffer with $\mathrm{Ca}^{2+} / \mathrm{Mg}^{2+}$, cell polarization became obvious, although no significant cell migration was detected (Fig. 1 A and Video S3). On ICAM-1/CXCL-12-coated surfaces, T lymphocyte polarization was more evident as seen by membrane ruffling at the leading edge and contraction of the opposing edge 


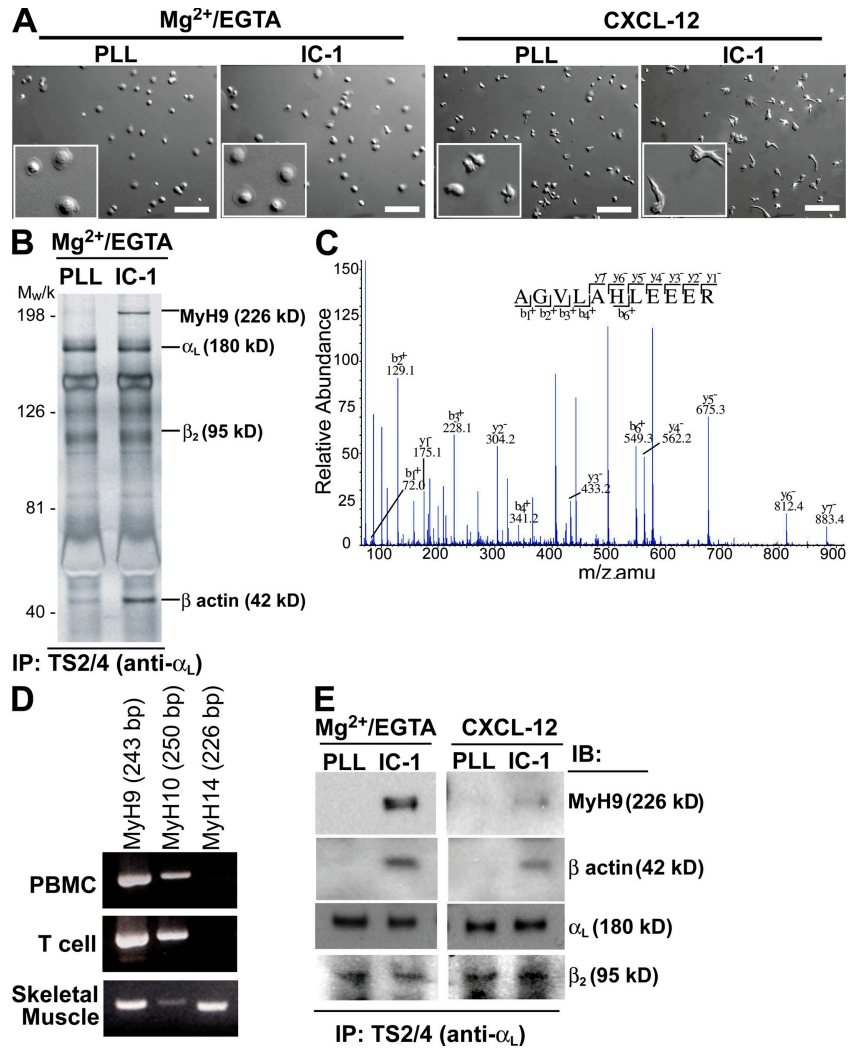

Figure 1. Association of MyH9 with LFA-1. (A) Human primary T lymphocytes were allowed to adhere to cover glasses coated with ICAM-1 (IC-1) or poly-L lysine (PLL) $\pm C X C L-12$. Cells were suspended in either L-15/2 mg/ml glucose or $20 \mathrm{mM}$ Hepes, $150 \mathrm{mM} \mathrm{NaCl}, 5 \mathrm{mM} \mathrm{MgCl}$, $1 \mathrm{mM}$ EGTA, and $2 \mathrm{mg} / \mathrm{ml}$ glucose, as indicated. Migrating T lymphocytes were tracked over a 20-min period, and time-lapse DIC images were acquired every $5 \mathrm{~s}$ to generate movies (Videos S1-4). The bottom left corner of each image shows a randomly selected region at threefold magnification. Bar, $100 \mu \mathrm{m}$. (B and C) LFA-1 immunoprecipitates obtained from bound cells (using TS2/4 antibody) were analyzed for possible LFA-1 binding partners. MyH9 was identified by silver staining $(n=3)(\mathrm{B})$ and mass spectrometry (C). The spectrum of one of the MyH9 peptides obtained by nanospray-ion trap tandem mass spectrometry is shown in C. (D) PCR amplification of $\mathrm{MyH} 9, \mathrm{MyH} 10$, and $\mathrm{MyH} 14$ cDNAs from human PBMCs and T lymphocytes. Reverse-transcribed cDNA from human skeletal muscle served as a positive control. (E) LFA-1 immunoprecipitates obtained for each of the four substrate conditions were subjected to Western blotting with the indicated antibodies.

to form the trailing edge (Fig. $1 \mathrm{~A}$ and Video S4). Unlike cells on poly-L lysine/CXCL-12 surface, these T lymphocytes actively migrated.

Analysis of LFA-1 immunoprecipitates from the adherent or migrating human $\mathrm{T}$ lymphocytes revealed that a prominent protein of $\sim 220 \mathrm{kD}$ was selectively associated with LFA-1 under conditions where Tlymphocytes were bound to ICAM-1 in the presence of $\mathrm{Mg}^{2+} /$ EGTA (Fig. $1 \mathrm{~B}$ ). This polypeptide could not be detected in LFA-1 immunoprecipitates obtained from poly-L lysine-bound T lymphocytes. The $220-\mathrm{kD}$ species was identified to be $\mathrm{MyH} 9$, as determined by mass spectrometry (Fig. 1 C). RT-PCR revealed that human T lymphocytes expressed both MyH9 and MyH10, but not MyH14 (Fig. 1 D). Mass spectrometric analysis of LFA-1 immunoprecipitates did not provide any evidence of an association between MyH10 and LFA-1. To confirm that the $220-\mathrm{kD}$ protein was, in fact, $\mathrm{MyH}$ 9, Western blot analysis with an anti-MyH9 antibody was performed. As can be seen in Fig. 1 E, the association of MyH9 with LFA-1 was most apparent in T lymphocytes bound to ICAM-1 in the presence of $\mathrm{Mg}^{2+} /$ EGTA, but it could also be seen in cells migrating on ICAM-1/CXCL-12 surfaces (Fig. 1 E). Notably, MyH9 association with LFA-1 always coincided with $\beta$-actin association (Fig. $1, \mathrm{~B}$ and $\mathrm{E}$ ).

\section{MyH9 mediates LFA-1 de-adhesion at the uropod}

To analyze the intracellular distribution of $\mathrm{MyH} 9$ during LFA-1-mediated T lymphocyte migration, primary human T lymphocytes were transfected with GFP-tagged MyH9 (MyH9GFP). Time-lapse fluorescence microscopy revealed that $\mathrm{MyH} 9$ was enriched in the uropod during migration, whereas $\beta$-actin was predominantly situated at the leading edge (Fig. $2 \mathrm{~A}$ and Videos S5 and S6, which are available at http://www .jem.org/cgi/content/full/jem.20071543/DC1). Although the uropodal distribution of $\mathrm{MyH} 9$ during migration hinted at a possible role for $\mathrm{MyH} 9$ in tail retraction, it was unclear if a direct relationship existed between $\mathrm{MyH} 9$ and LFA-1 deadhesion. To investigate this further, we used the highly specific inhibitor of class II myosin ATPase activity, blebbistatin $(28,29)$. Treatment of $T$ lymphocytes with blebbistatin prevented MyH9 association with LFA-1 (Fig. 2 D). Surprisingly, blebbistatin treatment resulted in a nonphysiological, extremely polarized cell morphology, in which the uropod was extended in length (Fig. 2, B and C, and Video S7). In stark contrast, the majority of control (DMSO-treated) cells were polarized with relatively short tails (Fig. 2, B and C, and Video S8).

The finding that blebbistatin induces extreme elongation of the uropod suggests that it may suppress $\mathrm{T}$ lymphocyte migration by preventing LFA-1 de-adhesion at the uropod. To test this hypothesis, we performed live-cell imaging of T lymphocyte migrating on ICAM-1/CXCL-12-coated glass surfaces. Quantitative analysis revealed that blebbistatin significantly decreased the lateral random migration of $\mathrm{T}$ lymphocytes on ICAM-1 (Fig. 2, E and F).

To confirm the results obtained with blebbistatin and to further assess the specificity of MyH9 in regulating LFA-1 deadhesion, small interfering RNA (siRNA)-mediated knockdown of $\mathrm{MyH} 9$ expression was performed. As shown in Fig. 3 C, siRNA selective for MyH9 (30) successfully reduced the protein expression level in human T lymphocytes. Similar to blebbistatin, MyH9 siRNA elicited abnormal uropodal extension (Fig. 3, A and B).

\section{MyH9 is not required for LFA-1 activation}

The observation that MyH9 is mainly localized at the uropod and mediates LFA-1 de-adhesion from ICAM-1 does not preclude its functioning in initial cell adhesion to ICAM-1 and in LFA-1 activation at the leading edge during cell migration. To test whether MyH9 association with LFA-1 


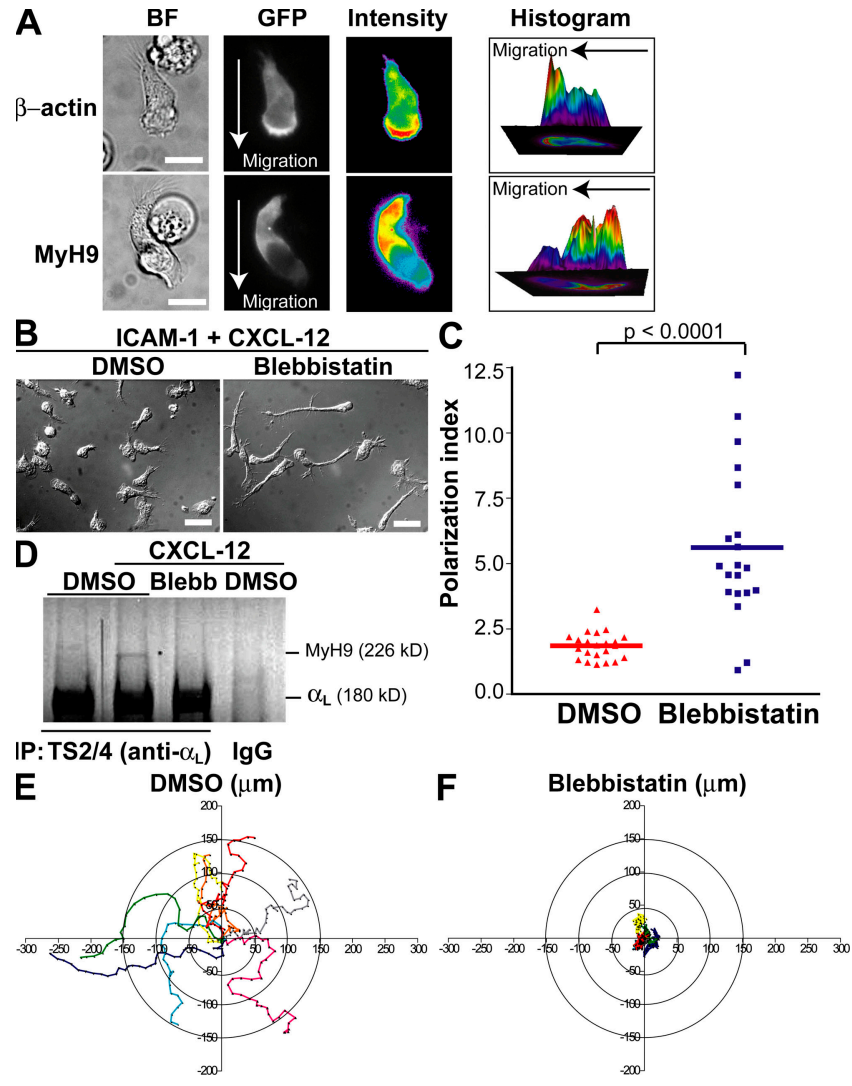

Figure 2. MyH9 mediates LFA-1 de-adhesion at the uropod. (A) Human T lymphocytes were transfected with MyH9-GFP or $\beta$-actin-GFP, and cell migration on ICAM-1 and CXCL-12 was analyzed by time-lapse fluorescence microscopy at $37^{\circ} \mathrm{C}$ (see also Video S5 for MyH9-GFP and Video $\mathrm{S} 6$ for $\beta$-actin-GFP). Arrows mark the direction of migration. Twodimensional images and three-dimensional histograms of fluorescence intensity and cell surface distribution are shown in a pseudo-color scale (from low [black] to high [red]). Bar, $20 \mu \mathrm{m}$. (B and C) Human T lymphocytes were pretreated with DMSO or $50 \mu \mathrm{M}$ blebbistatin and allowed to migrate on ICAM-1- and CXCL-12-coated cover glass for 20 min at $37^{\circ} \mathrm{C}$ (B). The polarization index of cells was calculated as the ratio of $x$ to $y$, where $x$ is the longest distance across cells (from head to tail) and $y$ is the greatest width perpendicular to $x$ (C). Bar, $25 \mu \mathrm{m}$ (B). (D) T lymphocytes were pretreated with DMSO or $50 \mu \mathrm{M}$ blebbistatin for $2 \mathrm{~h}$ and stimulated with CXCL-12 for 20 min. LFA-1 immunoprecipitates (TS2/4 antibody) were then obtained and subjected to silver staining. (E and F) Human T lymphocytes were pretreated with DMSO or $50 \mu \mathrm{M}$ blebbistatin for $1 \mathrm{~h}$ at $37^{\circ} \mathrm{C}$, and cell migration on ICAM-1/CXCL-12-coated cover glasses was tracked over a 30-min period. Each line represents one cell. Experiments were repeated on T lymphocyte preparations from three independent donors.

occurs before LFA-1 activation and possibly mediates LFA-1 activation, the kinetics of CXCL-12-induced LFA-1 activation were measured by flow cytometric analysis of KIM127 labeling. KIM127 is an activation-dependent monoclonal antibody; it recognizes a portion of the $\beta_{2}$ EGF2 domain that is buried in the bent, inactive conformation and exposed in the extended, active conformation. Unlike the continuous regeneration of active LFA-1 during cell migration, chemokines elicit transient LFA-1 activation in suspended cells $(31,32)$.
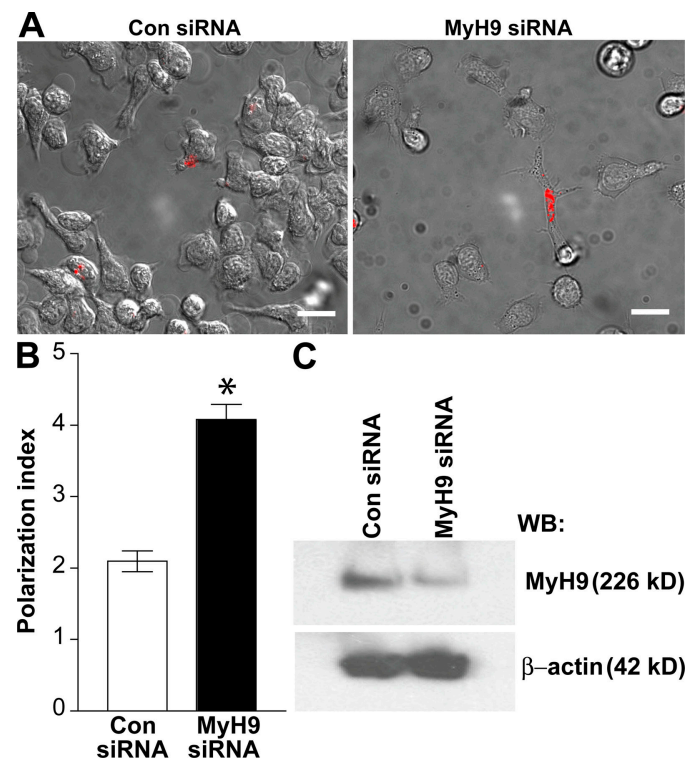

C

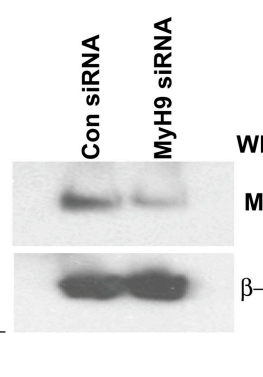

WB:

MyH9(226 kD)

$\beta-\operatorname{actin}(42 \mathrm{kD})$

Figure 3. Selective suppression of MyH9 protein with siRNA. Human primary T lymphocytes were transfected with Cy3-conjugated control siRNA only or Cy3-conjugated control siRNA plus MyH9 siRNA at a 1:10 ratio and allowed to migrate on ICAM-1/CXCL-12 cover glasses. (A) An overlay of DIC and Cy3 fluorescence images is shown. Bar, $20 \mu \mathrm{m}$. (B) Quantification of tail detachment, as determined by the polarization index. The polarization index was measured on $\mathrm{Cy} 3^{+}$cells. ${ }^{*}, \mathrm{P}<0.0001$ for $\mathrm{MyH} 9$ siRNA $(n=19)$ versus control siRNA only. (C) T lymphocyte cell lysates were analyzed for $\mathrm{MyH} 9$ and $\beta$-actin levels by Western blot. Experiments were repeated on T lymphocyte preparations from three independent donors.

Accordingly, binding of KIM127 to T lymphocytes in cell suspension significantly increased $5 \mathrm{~min}$ after adding CXCL-12, whereas the binding was undetectable at points beyond $20 \mathrm{~min}$ (Fig. 4 A). Rapid and transient LFA-1 activation was confirmed in cell adhesion assays, where T lymphocyte adhesion on immobilized ICAM-1 was significantly enhanced after 2 min of soluble CXCL-12 stimulation and rapidly down-regulated within 5-10 min (Fig. 4 B), and in a flow chamber assay, where $\mathrm{T}$ lymphocytes arrest on immobilized ICAM-1 and CXCL-12 triggered by LFA-1 activation occurs within $1 \mathrm{~s}$ under flow (Fig. 4 E). Therefore, stimulation of human primary T lymphocytes with CXCL-12 induces a rapid and transient LFA-1 activation that peaks within a few seconds after activation.

During rapid LFA-1 activation by CXCL-12, no significant association of MyH9 with LFA-1 was observed (Fig. 4 C). MyH9 and LFA-1 association occurred later, at $\sim 20 \mathrm{~min}$ after CXCL-12 stimulation (Fig. 4 C). All leukocytes that express LFA-1 also express one or more of its ligands, facilitating the formation of aggregates of homotypically adherent cells (16). Therefore, the late association of MyH9 with LFA-1 may be due to LFA-1 and ICAM-1 binding through homotypic T lymphocyte interaction in the cell suspension. Inhibition of MyH9 with blebbistatin had no effect on LFA-1 activation by CXCL-12 (Fig. 4, D and E). The results suggest that MyH9 recruitment to LFA-1 does not precede or coincide with LFA-1 activation. 
A $\underline{\text { mAb KIM127 }}$
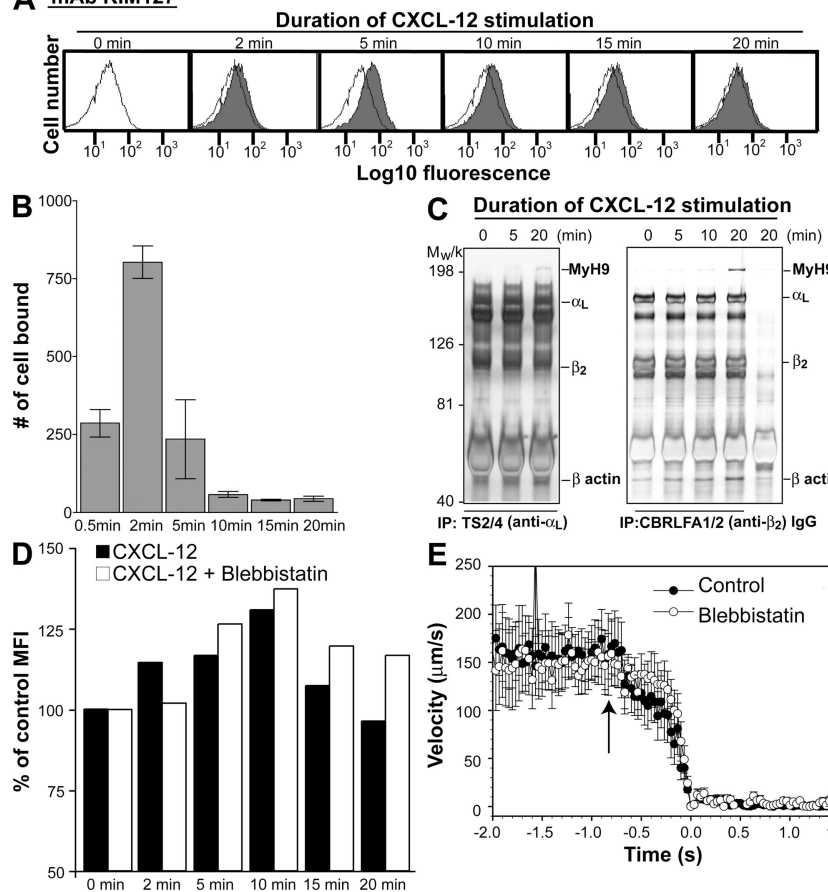

C Duration of CXCL-12 stimulation

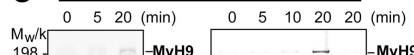
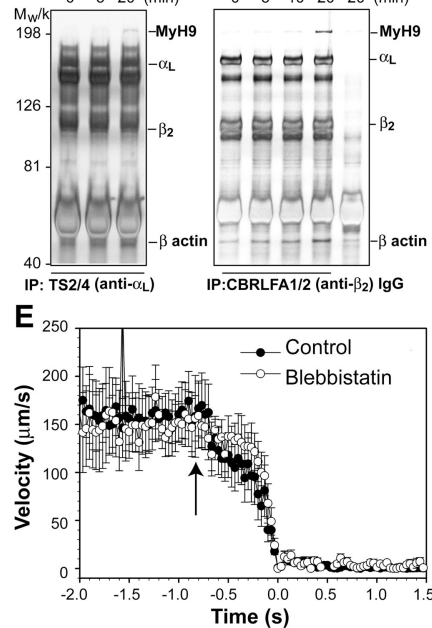

Figure 4. MyH9-LFA-1 association is not required for CXCL-12induced LFA-1 activation. (A) T lymphocytes were stimulated with (filled histograms) or without (open histograms) $100 \mathrm{ng} / \mathrm{ml} \mathrm{CXCL-12} \mathrm{for}$ the indicated time period. Cells were labeled with KIM127 antibody and subjected to flow cytometry. Experiments were performed in triplicate with cells from three different donors. Representative histograms showing the time course of KIM127 epitope expression are shown. (B) Human T lymphocytes adherent to ICAM-1-coated slides were stimulated with $100 \mathrm{nM}$ CXCL-12. Nonadherent cells were removed, and the number of bound cells was counted. Data are expressed as mean \pm SEM of three experiments. The experiment is representative of results from three independent donors. (C) Lysates obtained from CXCL-12-stimulated T lymphocytes were subjected to $\alpha_{\mathrm{L}}$ (TS2/4 antibody) and $\beta_{2}$ (CBR LFA-1/2 antibody) immunoprecipitation, and then to silver staining. Mouse $\lg G$ antibody served as a negative control. (D) T lymphocytes were pretreated with $50 \mu \mathrm{M}$ blebbistatin or DMSO and analyzed as described above (A). A representative result of three separate experiments is shown. (E) Data represent the mean instantaneous velocities of 10 representative T lymphocytes without (Control) or with $50 \mu \mathrm{M}$ blebbistatin (Blebbistatin), respectively, in a parallel-plate flow chamber coated with CXCL-12 and ICAM-1 at a wall shear stress of $0.5 \mathrm{dyn} / \mathrm{cm}^{2}$. T lymphocyte trajectory was monitored for $2 \mathrm{~s}$ before the first contact between cells and substrate (arrow) and arrest (time 0 ). The time interval between data points is $33 \mathrm{~ms}$. All data are expressed as mean \pm SEM.

The spatial regulation of LFA-1 activation is thought to play an important role in cell migration (33-35). Recently, the human monoclonal antibody AL-57, which specifically recognizes the active conformation of the $\alpha_{\mathrm{L}}$ I domain in a fashion that mimics physiological ligands, has been used to demonstrate the activation of LFA-1 on human T lymphocytes surface (32). We used this activation-dependent antibody to examine the effect of $\mathrm{MyH} 9$ inhibition with blebbistatin on the spatial distribution of active LFA-1 on polarized T lymphocytes during migration. A nonfunction blocking and activation-

insensitive monoclonal antibody, TS2/4, was used to measure total LFA-1. TS2/4 labeling revealed that LFA-1 molecules were broadly distributed on $\mathrm{T}$ lymphocytes migrating on ICAM-1 substrate in response to CXCL-12 (Fig. 5 A). AL-57 labeling was mainly present at the leading edge of migrating T lymphocytes under control conditions (Fig. $5 \mathrm{~A}$ ). The restricted distribution of AL-57 labeling at the leading edge was not significantly altered by blebbistatin treatment (Fig. 5 A).

Periodic lamellipodial contraction at the leading edge is often seen in spreading and migrating cells (36). We analyzed the periodic contraction of the protruding leading edge by creating kymographs using differential interference contrast (DIC) microscopy (Fig. 5 B). In control cells, a single cycle of the contraction, defined as a protrusion followed by a retraction (white arrows), had a period of $\sim 10 \mathrm{~s}$. The time interval between each contraction was not significantly altered by blebbistatin (Fig. $5 \mathrm{~B}$ and Video S9, which is available at http://www .jem.org/cgi/content/full/jem.20071543/DC1). These results, collectively with the finding that $\mathrm{MyH} 9$ association is not required for LFA-1 activation and blebbistatin does not affect localization of active high affinity LFA-1 at the leading edge of migrating $\mathrm{T}$ lymphocytes, suggest that $\mathrm{MyH} 9$ is dispensable for LFA-1 activation and de novo cell adhesion at the leading edge.

Although highly specific to myosin II, treatment of cells with $50 \mu \mathrm{M}$ blebbistatin only partially inhibits nonmuscle myosin heavy chain II functions (37). In addition, periodic retractions of the lamellipodia are induced by myosin light chain kinse-dependent, but Rho-associated kinase (ROCK)independent, force generation by myosin II (36). To investigate whether the contractile oscillations at the leading edge and the retraction at the uropod are differentially sensitive to blebbistatin, we tested a range of blebbistatin concentrations. Unlike a $50-\mu \mathrm{M}$ concentration, treatment of $\mathrm{T}$ lymphocytes with $200 \mu \mathrm{M}$ blebbistatin abolished the periodic lamellipodia retraction (Fig. 5 B) and dramatically reduced the uropod elongation (Fig. 5 C). Therefore, MyH9 at the uropod appears to be more susceptible to inhibition by blebbistatin than at the leading edge of migrating $\mathrm{T}$ lymphocytes.

\section{Affinity- and avidity-regulated LFA-1 mediates adhesion at the anterior and at the posterior of migrating T lymphocytes, respectively}

Both affinity regulation and avidity regulation govern the strength of integrin-ligand adhesiveness (38). The relative importance of the integrin activation states in cell migration remains unknown, but is thought to vary during the cell migration process. To investigate the spatial organization of affinity and avidity regulation of LFA-1 during T lymphocyte migration, we used two classes of LFA-1 small molecule antagonists, the $\alpha$ I allosteric antagonist, BIRT377, and the $\alpha / \beta$ I-like allosteric antagonist, XVA143. Because of their different modes of action (refer to Introduction), $\alpha$ I allosteric antagonists inhibit both affinity and avidity regulation of LFA-1, whereas $\alpha / \beta$ I-like allosteric antagonists inhibit only affinity regulation (16). 
A
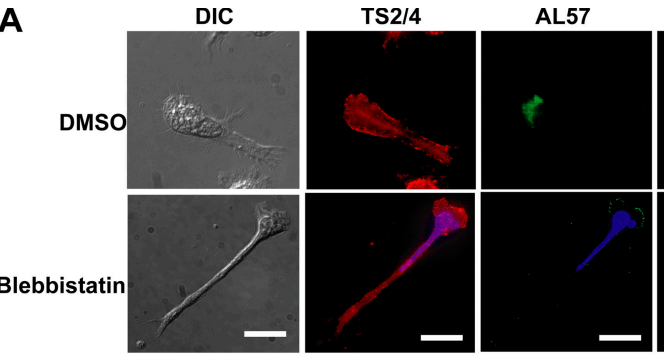

Combine

BDMSO
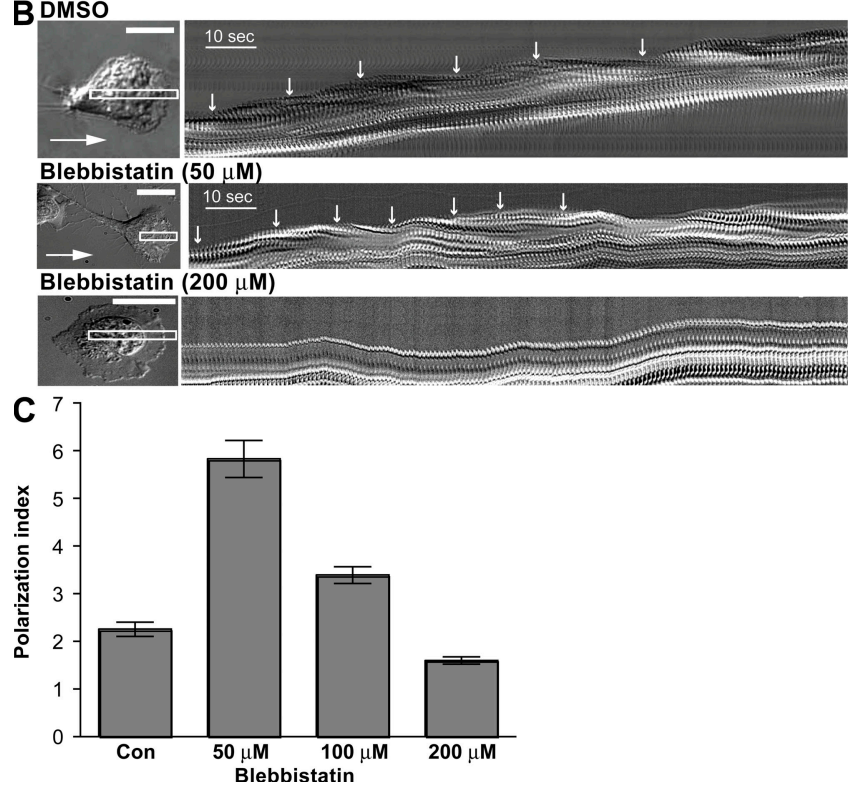

Figure 5. MyH9-LFA-1 association is not required for the localization of active LFA-1 at the leading edge. (A) Human primary T lymphocytes were pretreated for $1 \mathrm{~h}$ with DMSO or $50 \mu \mathrm{M}$ blebbistatin, incubated on cover glasses coated with ICAM-1 and CXCL-12 for 30 min, and processed for dual immunofluorescence labeling with AL-57 (green), an antibody specific for the active human $\alpha_{L}$ I domain, and TS2/4 (red). Nuclei of blebbistatin-treated cells were counterstained with DAPI (blue). Bar, $20 \mu \mathrm{m}$. (B) T lymphocytes treated with DMSO, $50 \mu \mathrm{M}$, or $200 \mu \mathrm{M}$ blebbistatin for $1 \mathrm{~h}$ were allowed to migrate on ICAM-1/CXCL-12-coated surfaces. DIC timelapse images were obtained at an acquisition rate of one frame per second. A narrow rectangular cursor was drawn on the time-lapse image stack (left, white line) so that the long axis of the rectangle was aligned with the direction of cell movement, which was determined by viewing the series as a movie. The rectangle was 4 pixels in width and long enough for complete motion analysis. This region of interest was then taken from each image in the time-lapse series, and the images were pasted side-by-side in a montage to form the kymograph picture. Each cycle of contractions was marked with white arrows (right). Bar, $10 \mu \mathrm{m}$. (C) T lymphocytes treated with DMSO (Con), $50 \mu \mathrm{M}, 100 \mu \mathrm{M}$, or $200 \mu \mathrm{M}$ blebbistatin for $1 \mathrm{~h}$ were allowed to migrate on ICAM-1/CXCL-12-coated surfaces. The polarization index of cells was calculated as the ratio of $x$ to $y$, where $x$ is the longest distance across cells (from head to tail) and $y$ is the greatest width perpendicular to x. 30 cells for each group were analyzed.

In control T lymphocytes, both BIRT377 and XVA143 (Video S11, available at http://www.jem.org/cgi/content/ full/jem.20071543/DC1) abolished cell polarization and inhibited adhesion to ICAM-1/CXCL-12 surfaces, suggesting that affinity regulation of LFA-1 is more critical in normal $\mathrm{T}$ lymphocyte migration (Fig. 6, A, B, and D). BIRT377 also blocked cell polarization and adhesion in the presence of blebbistatin (Fig. 6, A and D). In contrast, XVA143 was only able to reduce the total cell length by $\sim 50 \%$ in the presence of blebbistatin (Fig. 6 B). Time-lapse video microscopy revealed that XVA143 (Video S12) partially detached the front half of elongated $\mathrm{T}$ lymphocytes in the presence of blebbistatin, whereas BIRT377 (Video S11) fully detached the elongated T lymphocytes (Fig. 6 C). XVA143 also only partially reduced T lymphocyte binding to ICAM-1/CXCL-12 surfaces in the presence of blebbistatin (Fig. 6 D). These results suggest that LFA-1 affinity regulation mainly mediates cell adhesion at the anterior of the polarized $\mathrm{T}$ lymphocytes, whereas avidity regulation of LFA-1 predominantly mediates adhesion of the uropod.

To visualize the spatial organization of affinity regulation and avidity regulation at the cell-substrate contact, we performed total internal reflection fluorescence (TIRF) imaging of KIM127, m24, and TS2/4 labeling. Because the epitopes of
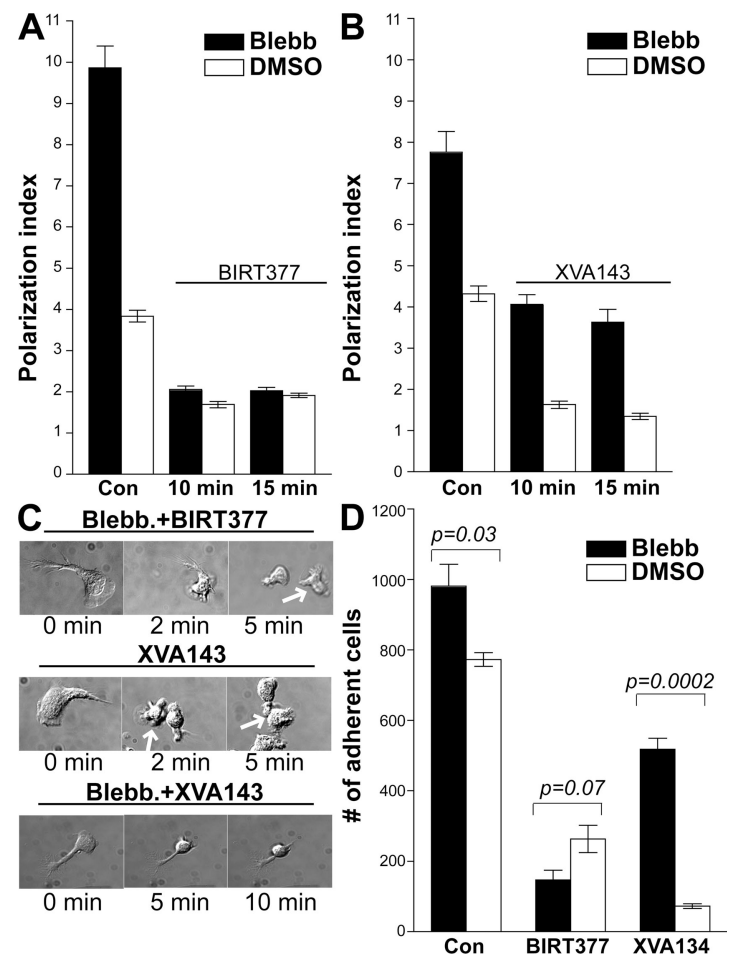

Figure 6. Uropodal adhesion depends on LFA-1 avidity regulation, and uropodal detachment is mediated by MyH9. (A-C) Human T lymphocytes were pretreated with DMSO or $50 \mu \mathrm{M}$ blebbistatin for $1 \mathrm{~h}$ and allowed to migrate on ICAM-1/CXCL-12-coated surfaces for $20 \mathrm{~min}$. Then cells were treated with $20 \mu \mathrm{M}$ BIRT377, $1 \mu \mathrm{M}$ XVA143, or an equivalent concentration of DMSO for the indicated times. The cell polarization index was then determined. Data are expressed as mean \pm SEM of three experiments, each performed in triplicate. Representative DIC images from time-lapse movies are shown in C. (D) T lymphocytes were pretreated with DMSO or blebbistatin and allowed to settle on the ICAM-1/CXCL-12coated cover glasses for $20 \mathrm{~min}$ in L-15 plus $2 \mathrm{mg} / \mathrm{ml}$ glucose. Cells were then treated with $20 \mu \mathrm{M}$ BIRT377, $1 \mu \mathrm{M}$ XVA143, or DMSO. After removal of nonadherent cells from the cover glasses, bound cells were counted. Data are expressed as mean \pm SEM of three experiments. 
KIM127 (39) and m24 (40) map to the EGF2 domain in the leg of $\beta_{2}$ subunit and the activated $\beta_{2}$ I-like domain, respectively, they will recognize both ligand-bound and -unbound active conformations of LFA-1 and thus, are predicted to detect affinity-regulated sites (41). As we predicted, KIM127 labeling was concentrated at the cell contact zone, predominantly at the anterior of the cell. Total LFA-1, as seen by labeling with the conformation nonselective antibody, TS2/4, was evenly distributed throughout the contact area (Fig. 7 A). Consistent with previous reports $(34,42)$, no obvious m24 labeling was displayed at the uropod (Fig. 7 B). These results suggest that LFA-1 at the posterior contact zone, which is not stained by KIM127 or m24, mediates cell adhesion without conformational activation, probably through avidity regulation.

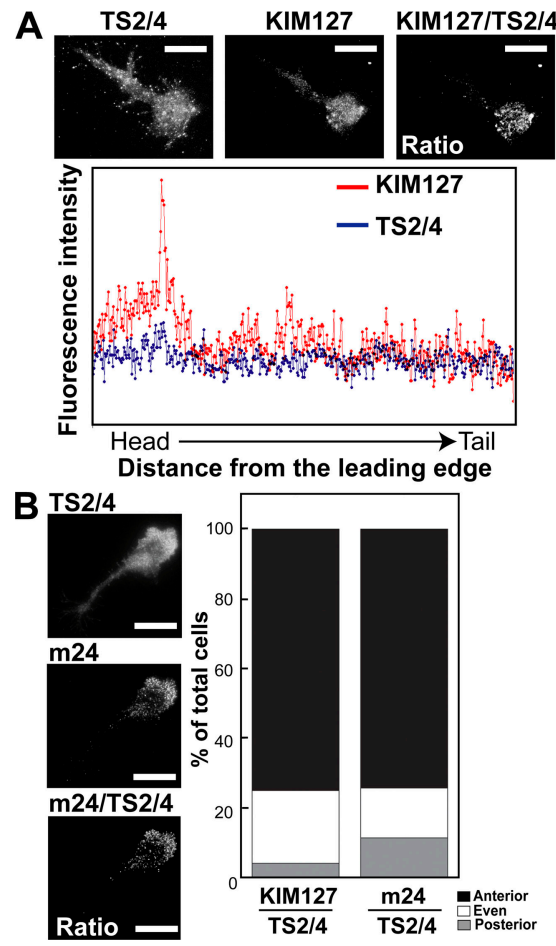

Figure 7. High affinity LFA-1 is not required for uropodal adhesion. ( $A$ and $B$ ) Human primary T lymphocytes were pretreated with blebbistatin and incubated on cover glasses coated with ICAM-1 and CXCL-12 for $20 \mathrm{~min}$. Cells were then processed for dual immunofluorescent labeling with TS2/4 antibody and KIM127 (A) or m24 (B) antibody and subjected to TIRF microscopy. Ratio images were generated by subtracting the background and dividing KIM127 (A) or m24 (B) intensity by TS2/4 intensity. Line profiles of LFA-1 intensity are presented along the front (Head)-to-back (Tail) line (A). (B) From three independent experiments, a total of 48 (KIM127) TS2/4) and 35 (m24/TS2/4) cells was randomly selected, carefully analyzed, and scored for the presence of KIM127 or m24 staining enriched at the anterior region, the posterior region, or both, based on the ratio images. Each bar represents the percentage of total cells scored. The black portion of each bar is the fraction of cells scored for the anterior region dominant staining of KIM127 or m24. The white portion of each bar is the fraction of the cells that showed even distribution of staining. The gray portion of each bar is the fraction of cells scored for the posterior region dominant staining of KIM127 or m24. Bar, $20 \mu \mathrm{m}$.

\section{DISCUSSION}

The data presented here demonstrate that $\mathrm{MyH} 9$ associates with ICAM-1-bound LFA-1 in T lymphocytes and provides the critical force for rapid rupture of the bonds between LFA-1 and ICAM-1 at the uropod during cell migration. MyH9 association with LFA-1 is mainly involved in the tail detachment from the ICAM-1 surface during migration, but is not directly related to LFA-1 activation. The overall strength of cellular adhesive interactions results from the combination of both the affinity of individual receptor-ligand bonds and the total number of bonds formed. According to Bell's theory (43), although most individual cellular bonds are reversible, a mechanical force against a bond is required to separate the cells when many bonds exist between them ("avidity regulation"), and the mechanical force acting against a bond can increase the frequency of bond dissociation between the receptor and the bound ligand. Thus, as a cell migrates, its ability to detach its tail from the posterior contacts may depend on the cytoskeletal contractile force exerted against the bonds between the plasma membrane adhesion molecules and their ligands. Our findings support this theory and demonstrate that, as T lymphocytes migrate on ICAM-1 surfaces, the contact between the tail and the substrate is mediated by the "valency" of the interaction between low affinity LFA-1 and ICAM-1, and that subsequent detachment depends on the contractile force exerted by $\mathrm{MyH} 9$ on LFA-1.

$\mathrm{MyH} 9$ is one of three different isoforms of the heavy chain of type II nonmuscular myosin. Although MyH9 is clearly involved in cell migration and there have been many reports of myosin II localization at the posterior of migrating cells (44), no studies have assessed the specific role of MyH9 in integrinmediated cell adhesion and migration. Consistent with our findings in human $\mathrm{T}$ lymphocytes, a recent study in mouse lymphocytes has revealed that $\mathrm{MyH} 9$ is localized to the uropod (45). In addition, inhibition of $\mathrm{MyH} 9$ dramatically reduced $\mathrm{T}$ lymphocyte motility and migration. The authors proposed that $\mathrm{MyH} 9$ inhibition reduces cell migration mainly by abolishing cell polarization. In stark contrast, our study reveals that inhibition of MyH9 in human T lymphocytes induces an extreme polarization as well as a defect in tail retraction, with the motility of the leading edge being only minimally affected. This discrepancy may be due to the different sensitivity of mouse MyH9 to blebbistatin (Fig. 5 C) or the different substrates used. In the aforementioned study, migration assays were performed mainly on plain cover glass, which lacks protein substrates required for cell migration. On the other hand, most of our experiments have been performed on ICAM-1, which is a highly specific ligand for LFA-1.

The association between LFA-1 and MyH9 was demonstrated by coimmunoprecipitation of endogenous proteins. This association occurs in response to LFA-1 binding to ICAM-1, but not in response to CXCL-12 stimulation. A direct association between myosin and integrin has previously been reported (46). Myo10, an unconventional myosin, binds directly to the NPXY motif of integrin $\beta$ subunit cytoplasmic domains via its FERM (band 4.1/ezrin/radixin/moesin) 
domain (46). Overexpression of Myo10 results in the formation of elongated filopodia due to the transport of excess integrins to the tips of filopodia. Unlike Myo10, MyH9 does not contain any known integrin-binding motifs in its tail domain. Therefore, it is possible that association of $\mathrm{MyH} 9$ with LFA-1 may require adaptor molecules. Interestingly, the LFA-1-MyH9 complex also contains $\beta$-actin (Fig. 1 B). Although it is currently unclear whether there are additional proteins in the complex, it is tempting to speculate that $\beta$-actin binds to LFA-1 through adaptor molecules such as talin $(9,47,48)$, and activated MyH9 binds to the LFA-1$\beta$-actin complex to exert the mechanical force necessary for LFA-1 detachment.

Blebbistatin was originally discovered in a screen for inhibitors of nonmuscle myosin II (28). Blebbistatin, a noncompetitive myosin II inhibitor, functions by binding the large cleft in the motor domain, which opens and closes during the contractile cycle. Binding of blebbistatin to myosin II results in a long-lived complex of myosin with ADP and inorganic phosphate. The formation of this complex precedes the forcegenerating step, which is catalyzed by the release of phosphate upon the rebinding of myosin with actin. Thus, blebbistatin inhibits the transition into force-producing states (49). Therefore, the elongated tail and the defects in tail detachment seen in blebbistatin-treated T lymphocytes on ICAM-1 may simply be due to down-regulation of the cytoskeletal traction force that is required for tail contraction. The concentration of blebbistatin used here $(50 \mu \mathrm{M})$ did not totally abolish tail contraction; however, BIRT377 co-treatment caused the elongated T lymphocytes to quickly contract their tails and round up (Fig. 6, A and C, and Video S10). These results suggest the presence of both a blebbistatin-resistant cellular contraction force and a blebbistatin-sensitive LFA-1 detachment force at the posterior of migrating $\mathrm{T}$ lymphocytes.

Surface particle tracking measurements on wild-type and mutant Dictyostelium lacking myosin II suggest that a myosin II-based force predominates at the posterior of the cell (50). Myosin II-mediated cell contractility is also implicated in adhesion, protrusion, and actin organization at the leading edge, especially in slow migrating cells (51). There are numerous reports that myosin II contraction is a key regulator of adhesion maturation and actin organization at the anterior of cells $(51,52)$. Compared with the dramatic effects of $50 \mu \mathrm{M}$ blebbistatin at the tail of migrating $\mathrm{T}$ lymphocytes, the effects of myosin II inhibition at the leading edge require higher concentrations of blebbistatin (Fig. 5 and Video S9). Based on this, we conclude that, in fast migrating $\mathrm{T}$ lymphocytes, the cellular contraction force generated at the anterior of the cell is less sensitive to blebbistatin, and that $\mathrm{MyH} 9$ provides a blebbistatin-dependent detachment force at the tail.

Inhibition of ROCK in human $\mathrm{T}$ lymphocytes resulted in similar cell morphology as we have seen in our T lymphocyte migration assay in the presence of blebbistatin, showing extreme tail elongation (53). Like the inhibition of $\mathrm{MyH}$, ROCK inhibition had no effect on the T lymphocyte adhesion on ICAM-1. Involvement of ROCK activity in the tail detachment of $\mathrm{T}$ lymphocytes was further confirmed by inhibition of RhoA, which is an upstream activator of ROCK (53). Phosphorylation of the myosin regulatory light chain (MLC) stimulates the actin-dependent ATPase activity of myosin II and promotes the filament assembly. At least three kinases can phosphorylate MLC in vitro: myosin light chain kinase, ROCK, and p21-activated kinase (24). Unlike other kinases, ROCK is mainly localized at the trailing edge during $\mathrm{T}$ lymphocyte migration, suggesting that it may play a major role in the signaling events responsible for $\mathrm{MyH} 9$ activation and LFA-1 de-adhesion. Therefore, it is tempting to speculate that LFA-1 outside-in signaling mediated by the RhoAROCK pathway (54) may impinge on $\mathrm{MyH} 9$ to regulate LFA-1 de-adhesion during T lymphocyte migration. Direct functional relation between MyH9 and ROCK in T lymphocytes is not currently known, but RhoA and its effector kinase ROCK regulate MyH9 activity through MLC phosphorylation in platelet (55).

We have previously found (16) that $\alpha$ I- (BIRT377) and $\alpha / \beta$ I-like (XVA143) allosteric antagonists provide a useful means for distinguishing between affinity and avidity regulation of LFA-1 adhesiveness. Using these two classes of antagonists, we have demonstrated that LFA-1 affinity regulation occurs at the anterior of the polarized T lymphocyte, and LFA-1 avidity regulation mainly works at the posterior to mediate cell adhesion during migration. The ability of XVA143 to inhibit $\mathrm{T}$ lymphocyte migration on ICAM-1 demonstrates that affinity regulation of LFA-1 is crucial for normal cell migration. In contrast, the inability of XVA143 to restore tail detachment in the presence of blebbistatin demonstrates that LFA-1-ICAM-1 interactions at the tail of migrating T lymphocytes are not subject to affinity regulation.

The lack of affinity regulation (active conformation) of LFA-1 at the tail adhesion was also confirmed by TIRF microscopy of KIM127 and m24 labeling. Binding of ligands to the extracellular domains of integrins initiates intracellular signaling (outside-in signal) and induces conformational changes in both the extracellular and the cytoplasmic domains. These conformational changes are predicted to be similar to those induced by inside-out signals. Therefore, because KIM127 and $\mathrm{m} 24$ recognize the EGF2 domain in the leg of the $\beta_{2}$ subunit and the activated $\beta_{2}$ I-like domain, respectively, it is also likely that they recognize both ligand-bound and -unbound active conformations of LFA-1. Both ligand-bound and -unbound active LFA-1, as identified by KIM127 and m24, are primarily located at the anterior of the cell body, but less at the posterior. Also, the $\alpha_{\mathrm{L}} \mathrm{I}$ domain antibody AL-57 revealed that a small population of ICAM-1-unoccupied active LFA-1 is exclusively located at the tip of the leading edge. Collectively, our results support the hypothesis that high affinity LFA-1 forms a stable adhesion in the front area of migrating $\mathrm{T}$ lymphocytes and switches to a low affinity conformation upon reaching the uropod, where it maintains adhesion through avidity regulation. Moreover, $\mathrm{MyH} 9$ is critical for generating sufficient pulling force to detach LFA-1 from ICAM-1 at the tail and to retract the uropod. 


\section{MATERIALS AND METHODS}

Materials. The small molecule LFA-1 inhibitor XVA143 was provided by P. Gillespie (Roche). The inhibitor BIRT377 was from Boehringer Ingelheim Pharmaceuticals Inc. The source for anti-human $\alpha_{\mathrm{L}}$ monoclonal antibody TS2/4 has been described previously (39). The monoclonal antibody m24 was provided by N. Hogg (London Research Institute, London, UK). KIM127 monoclonal antibody was prepared from hybridoma (American Type Culture Collection). Monoclonal antibody to $\beta$-actin was from Abcam, and $\mathrm{MyH} 9$ antibody was from Covance. Blebbistatin was purchased from Sigma-Aldrich.

Cell culture. PBMCs were separated from $50 \mathrm{ml}$ of whole blood by Histopaque (Sigma-Aldrich) density gradient centrifugation. Lymphocytes were cultured for $3 \mathrm{~d}$ in RPMI 1640 containing $10 \% \mathrm{FBS}$ and $1 \mu \mathrm{g} / \mathrm{ml}$ PHA (Remel). Lymphocytes were then cultured in the presence of $10 \mathrm{ng} / \mathrm{ml} \mathrm{IL-15}$ (R\&D Systems) for $4-7 \mathrm{~d}$. These cells were $97 \% \mathrm{CD}^{+}$and $\mathrm{CD} 56^{-}$as determined by flow cytometry (16). The Human Research Studies Review Board of the University of Rochester approved this study.

RT-PCR. Total RNA was prepared from PBMCs and a T lymphocyte RNeasy mini kit (QIAGEN). Isolated mRNA was reverse transcribed and amplified by PCR in one step using a QIAGEN One-Step RT-PCR kit with the following sense and antisense primers: $\mathrm{MyH} 9$ : sense, gaagagctagaggcgctgaa; antisense, ctttgccttctcgaggtttg; MyH10: sense, gtaccttgcccatgttgctt; antisense, ttttgcttgacgaacagcac; and $\mathrm{MyH} 14$ : sense, ccagaatcgggaaagtgaaa; antisense, ctcctcctccagctcttcct.

siRNA. A pool (SMARTpool) of siRNAs specific for human MyH9 and Cy3-conjugated RISC-free control siRNAs were synthesized by Dharmacon. Primary T lymphocytes were transfected using the AMAXA Nucleofector system (Program T-14). Cultures were transfected with $0.15 \mu \mathrm{g}$ Cy3-conjugated RISC-free control siRNA $\pm 1.45 \mu \mathrm{g} \mathrm{MyH} 9$ siRNA. After transfection, cells were cultured for $18 \mathrm{~h}$ in IL-15-containing media (RPMI 1640, 10\% FBS, $10 \mathrm{ng} / \mathrm{ml} \mathrm{IL-15).} \mathrm{Cy3} \mathrm{fluorescent} \mathrm{cells} \mathrm{were} \mathrm{considered} \mathrm{to}$ be MyH9 siRNA cotransfectants.

FACS analysis. $2 \times 10^{6}$ primary T lymphocytes were treated with $50 \mu \mathrm{M}$ blebbistatin or a 1:1,000 dilution of DMSO for $1 \mathrm{~h}$ at $37^{\circ} \mathrm{C}$, and then stimulated with $100 \mathrm{ng} / \mathrm{ml} \mathrm{CXCL-12} \mathrm{(R \& D} \mathrm{Systems)} \mathrm{for} 0,2,5,10,15$, or $20 \mathrm{~min}$. Cells were fixed with an equal volume of $2 \times$ formaldehyde (7.4\%) and incubated with $4 \mu \mathrm{g} / \mathrm{ml} \mathrm{KIM} 127$ antibody for $30 \mathrm{~min}$. After thorough washing, cells were then incubated with R-phycoerythrin-conjugated donkey anti-mouse IgG antibody (1:100; BD Biosciences). Mean fluorescence intensity was determined using a Becton Dickinson FACS Array system and WinMDI 2.8 software.

Immunoprecipitation. Cover glasses were coated with $6 \mu \mathrm{g} / \mathrm{ml}$ of human tonsil ICAM-1 or $10 \mu \mathrm{g} / \mathrm{ml}$ poly-L lysine in the presence or absence of $2 \mu \mathrm{g} / \mathrm{ml}$ CXCL-12 (suspended in $20 \mathrm{mM}$ Tris, $\mathrm{pH} 9,159 \mathrm{mM} \mathrm{NaCl}$, and $2 \mathrm{mM} \mathrm{MgCl}$ ). Coatings were performed at $4{ }^{\circ} \mathrm{C}$ for $12 \mathrm{~h}$, followed by $4 \mathrm{~h}$ at room temperature. T lymphocytes $\left(5 \times 10^{6} / 500 \mu \mathrm{l}\right)$ were washed and resuspended in L-15 medium (Invitrogen) containing $2 \mathrm{mg} / \mathrm{ml}$ glucose or in $20 \mathrm{mM}$ Hepes, $150 \mathrm{mM}$ $\mathrm{NaCl}, 5 \mathrm{mM} \mathrm{MgCl}_{2}, 1 \mathrm{mM}$ EGTA, and $2 \mathrm{mg} / \mathrm{ml}$ glucose. They were then incubated at $37^{\circ} \mathrm{C}$ for $20 \mathrm{~min}$ on the coated cover glass. Cells were lysed in cold buffer containing HBSS, 2\% Triton X-100, $120 \mathrm{mM} \mathrm{N-octyl} \beta$-D-glucopyranoside, and EDTA-free mini-protease inhibitor cocktail (Roche). Insoluble cell debris was removed by centrifugation. Lysates were then precleared with $2 \mu \mathrm{g}$ mouse $\operatorname{IgG}$ isotype control antibody and protein $\mathrm{G}$ agarose beads (Invitrogen) for $30 \mathrm{~min}$ at $4^{\circ} \mathrm{C}$. Immunoprecipitation was performed by incubating lysates with protein $\mathrm{G}$ agarose and $14 \mu \mathrm{g}$ TS2/4 or CBR LFA1/2 antibody for $2 \mathrm{~h}$ at $4^{\circ} \mathrm{C}$. Beads were thoroughly washed, suspended in $30 \mu \mathrm{l}$ Laemmli buffer, and boiled for $5 \mathrm{~min} .5 \mu \mathrm{l}$ of each sample was separated on a 4-12\% gradient gel (Cambrex), and the gel was silver stained using a Silver Quest kit (Invitrogen). Species of interest were excised, destained, and analyzed by mass spectrometry. For immunoblotting, proteins were transferred to
PVDF membrane (Bio-Rad Laboratories). Membranes were probed with primary antibodies and appropriate peroxidase-conjugated secondary antibody. Immunoreactive bands were visualized by chemiluminescence.

Migration assay. Delta $\mathrm{T}$ dishes (Thermo Fisher Scientific) were coated with $6 \mu \mathrm{g} / \mathrm{ml}$ of human tonsil ICAM-1 and $2 \mu \mathrm{g} / \mathrm{ml} \mathrm{CXCL-12} \mathrm{(suspended}$ in $20 \mathrm{mM}$ Tris, $\mathrm{pH} 9,159 \mathrm{mM} \mathrm{NaCl}$, and $2 \mathrm{mM} \mathrm{MgCl}_{2}$ ) for $12 \mathrm{~h}$ at $4^{\circ} \mathrm{C}$ and for $4 \mathrm{~h}$ at room temperature. To inhibit $\mathrm{MyH}$, primary $\mathrm{T}$ lymphocytes were incubated with $50 \mu \mathrm{M}$ blebbistatin for $1 \mathrm{~h}$ or transfected with MyH9 siRNA. Either DMSO $(1: 1,000)$ or nontargeting siRNA served as a control. Approximately $5 \times 10^{4} \mathrm{~T}$ lymphocytes were washed and added to the delta $\mathrm{T}$ dish containing $1 \mathrm{ml}$ of L-15 medium with $2 \mathrm{mg} / \mathrm{ml}$ glucose \pm blebbistatin. Bright field or DIC images were acquired every second or every $5 \mathrm{~s}$ for $30 \mathrm{~min}$ under a $20 \times$ objective lens using MetaVue software. The temperature was maintained at $37^{\circ} \mathrm{C}$ throughout the experiment. Migration was analyzed by tracing the movement of individual cells in video using Image J software.

Assay of cell adhesion to ICAM-1. In vitro dynamic cell adhesion assays were performed as reported previously $(56,57)$. In brief, cells were resuspended at $10^{7} / \mathrm{ml}$ in L-15 medium containing $2 \mathrm{mg} / \mathrm{ml}$ glucose. Adhesion assays were performed on 18-well glass slides. Slides were coated with purified human tonsil ICAM-1 $(1 \mu \mathrm{g} / \mathrm{ml}$ in $20 \mathrm{mM}$ Tris, $\mathrm{pH}$ 9, $150 \mathrm{mM} \mathrm{NaCl}$, and $2 \mathrm{mM} \mathrm{MgCl}$ ) overnight at $4^{\circ} \mathrm{C}$ and for $4 \mathrm{~h}$ at room temperature. They were then blocked with $2 \% \mathrm{BSA}$ at $37^{\circ} \mathrm{C} .5 \mu \mathrm{l}$ of cell suspension was added to each well, and cells were stimulated at $37^{\circ} \mathrm{C}$ with $5 \mu$ of $200 \mathrm{nM} \mathrm{CXCL-12.} \mathrm{Cells}$ were then washed and fixed, and the number of bound cells was quantified.

Flow chamber assay was performed in a rectangular parallel-plate flow chamber (GlycoTech) with a gasket $(0.25 \mathrm{~cm}$ wide, $127 \mathrm{~mm}$ thick, and $2 \mathrm{~cm}$ long). Polystyrene Petri dishes (diameter, 35 mm; Falcon 1008) enclosed using a single-well flexiPERM (Sigma-Aldrich) were coated with a mixed solution of $10 \mu \mathrm{g} / \mathrm{ml}$ Purified Recomb Protein A/G (Thermo Fisher Scientific) and $1 \mu \mathrm{g} / \mathrm{ml} \mathrm{CXCL-12}$ at room temperature for $2 \mathrm{~h}$. The surfaces were then washed with $1 \%$ BSA in PBS. $5 \mu \mathrm{g} / \mathrm{ml}$ ICAM-1/Fc (R\&D Systems) was applied to the surfaces and allowed to bind the protein $A / G$-coated surfaces at room temperature for $2 \mathrm{~h}$. The surfaces were then blocked for nonspecific adhesion with $2 \% \mathrm{BSA}$ in PBS at $4^{\circ} \mathrm{C}$ for overnight. Before the flow chamber experiments, the dishes were washed with prewarmed L-15 medium $\left(37^{\circ} \mathrm{C}\right)$. The flow chamber was mounted on an inverted microscope (IX81; Olympus) and a microscope stage top incubator (LiveCell2; Pathology Devices) was installed on the flow chamber to perform the flow chamber experiments at $37^{\circ} \mathrm{C} .10^{6} / \mathrm{ml} \mathrm{T}$ lymphocytes were prewarmed in $\mathrm{L}-15$ medium at $37^{\circ} \mathrm{C}$ and perfused into the chamber using a syringe pump (New Era Pump Systems Inc.) at a flow rate corresponding to a calculated wall shear stress of $0.5 \mathrm{dyn} / \mathrm{cm}^{2}$. To investigate the effect of blebbistatin, T lymphocytes were incubated with $50 \mu \mathrm{M}$ blebbistatin at $37^{\circ} \mathrm{C}$ for $1 \mathrm{~h}$ before experiments. Arrest of T lymphocytes on immobilized ICAM-1 was observed in the field of view of $864 \times 648 \mu \mathrm{m}^{2}$ using bright field microscopy under a $10 \times$ objective (NA, 0.30; Plan Fluorite; Olympus) with a microscope-linked CCD camera (KPM1AN; Hitachi). Cell adhesion videos were redigitalized to $640 \times 480$ pixels at $29.97 \mathrm{fps}$ with ffmpegX software. The stacked images were thresholded and de-interlaced, and the coordinates of the centroid of interacting $\mathrm{T}$ lymphocytes with immobilized ICAM-1 were then acquired using Image J 1.39a (National Institutes of Health [NIH]). To overlay the cell trajectories, the earliest time when each cell rolled slower than $5 \mu \mathrm{m} / \mathrm{s}$ was set to zero.

Fluorescence microscopy. Coverslips were prepared as described for immunoprecipitation experiments. T lymphocytes were washed, resuspended in L-15 medium containing $2 \mathrm{mg} / \mathrm{ml}$ glucose, and incubated on the cover glass for $20 \mathrm{~min}$ at $37^{\circ} \mathrm{C}$. Cells were transferred to room temperature, fixed (3.7\% formaldehyde in PBS for $10 \mathrm{~min}$ ), and blocked with 10\% FBS overnight. Cells were labeled with $10 \mu \mathrm{g} / \mathrm{ml} \mathrm{AL}-57 \mathrm{IgG}$ for $1 \mathrm{~h}$, followed by a 1 -h incubation with $10 \mu \mathrm{g} / \mathrm{ml}$ anti- $\alpha_{\mathrm{L}}$ monoclonal antibody (TS2/4). Cells were then incubated with Cy3-conjugated anti-mouse Ig (Invitrogen) and FITC-conjugated anti-human Ig (Invitrogen) for $20 \mathrm{~min}$, and then washed three times with PBS. Image acquisition was conducted on an epifluorescence 
microscope (TE2000-U microscope; Nikon), using a $60 \times$ oil objective coupled to a CoolSNAP HQ CCD (Roper Scientific).

TIRF microscopy. TIRF imaging was performed with a white light TIRF aperture diaphragm coupled to a microscope (TE2000-S; Nikon) and a 100X TIRF 1.49 NA oil immersion objective. All image processing was performed with MetaVue and Metamorph software (Molecular Devices). Cellular staining was performed as described above using $10 \mu \mathrm{g} / \mathrm{ml}$ of monoclonal antibody fluorescent conjugates, KIM127 Alexa 546, and TS2/4 Alexa 488. Alternatively, cells were stained using $10 \mu \mathrm{g} / \mathrm{ml}$ of $\mathrm{m} 24$ antibody in conjunction with Cy-3 goat anti-mouse IgG and TS2/4 Alexa 488.

Online supplemental material. In Video S1, time-lapse video microscopy of human $\mathrm{T}$ lymphocyte migration on poly-L-lysine in the presence of $\mathrm{Mg}^{2+} /$ EGTA (1 sec video $=2.5 \mathrm{~min}$ real time) is shown. Video S2 features time-lapse video microscopy of human $\mathrm{T}$ lymphocyte migration on ICAM-1 in the presence of $\mathrm{Mg}^{2+} /$ EGTA ( $1 \mathrm{sec}$ video $=2.5 \mathrm{~min}$ real time). Video S3 shows time-lapse video microscopy of human $\mathrm{T}$ lymphocyte migration on poly-L-lysine/CXCL-12 surface $(1 \mathrm{sec}$ video $=2.5 \mathrm{~min}$ real time). In Video S4, time-lapse video microscopy of human $\mathrm{T}$ lymphocyte migration on ICAM-1/CXCL-12 surface ( $1 \mathrm{sec}$ video $=2.5 \mathrm{~min}$ real time) is shown. In Video S5, a time-lapse movie shows GFP intensity (highest [red] to lowest [blue]) of a MyH9-GFP-transfected human T lymphocyte migrating on ICAM-1/CXCL-12. The time scale of each frame is $30 \mathrm{sec}$. Video S6 is a time-lapse movie showing GFP intensity (highest [red] to lowest [blue]) of a $\beta$ actin-GFP-transfected human T lymphocyte migrating on ICAM-1/CXCL-12. The time scale of each frame is $30 \mathrm{sec}$. Videos S7 and S8 feature time-lapse video microscopy of a blebbistatin (50 $\mu \mathrm{M}$; Video S7) or DMSO (1:1,000 dilution; Video S8) -treated human T lymphocyte migrating on ICAM-1/CXCL-12 surface (1 sec video $=2.5 \mathrm{~min}$ real time). Video $S 9$ shows a high-resolution $(60 \times$ objective $+1.5 \times$ zoom $)$ movie of a blebbistatin $(50 \mu \mathrm{M})$-treated human $\mathrm{T}$ lymphocyte migrating on ICAM-1/CXCL-12 surface. Time scale of each frame is $1 \mathrm{sec}$. In Video S10, time-lapse video microscopy shows inhibition of $\mathrm{T}$ lymphocyte migration on ICAM-1/CXCL-12 by XVA143 (1 sec video $=2.5$ min real time). XVA143 was added during the interval of the movie. In Video S11, timelapse video microscopy shows inhibition of a blebbistatin-treated human $\mathrm{T}$ lymphocyte migrating on ICAM-1/CXCL-12 by BIRT377 (1 sec video = 2.5 min real time). BIRT377 was added during the interval of the movie. In Video S12, time-lapse video microscopy shows partial inhibition of a blebbistatin-treated human T lymphocyte migrating on ICAM-1/CXCL-12 by XVA143 (1 sec video $=2.5$ min real time). XVA143 was added during the interval of the movie. Videos S1-S12 are available at http://www.jem .org/cgi/content/full/jem.20071543/DC1.

This project was supported by the American Heart Association (to M. Kim), the Rhode Island Foundation (to M. Kim), NIH HL087088 (to M. Kim), NIH GM066194 (to J.S. Reichner), National Science Foundation DMR 0079964 (to J.X. Tang), and Brown University Seed Grant (J.X. Tang).

The authors have no conflicting financial interests.

Submitted: 25 July 2007

Accepted: 5 December 2007

\section{REFERENCES}

1. Semmrich, M., A. Smith, C. Feterowski, S. Beer, B. Engelhardt, D.H. Busch, B. Bartsch, M. Laschinger, N. Hogg, K. Pfeffer, and B. Holzmann. 2005. Importance of integrin LFA-1 deactivation for the generation of immune responses. J. Exp. Med. 201:1987-1998.

2. Vinogradova, O., A. Velyvis, A. Velyviene, B. Hu, T.A. Haas, E.F. Plow, and J. Qin. 2002. A structural mechanism of integrin $\alpha_{\mathrm{IIb}} \beta_{3}$ "inside-out" activation as regulated by its cytoplasmic face. Cell. 110:587-597.

3. Calderwood, D.A., R. Zent, R. Grant, D.J. Rees, R.O. Hynes, and M.H. Ginsberg. 1999. The talin head domain binds to integrin beta subunit cytoplasmic tails and regulates integrin activation. J. Biol. Chem. 274:28071-28074.
4. Calderwood, D.A., B. Yan, J.M. de Pereda, B. Garcia-Alvarez, Y Fujioka, R.C. Liddington, and M.H. Ginsberg. 2002. The phosphotyrosine binding (PTB)-like domain of talin activates integrins. J. Biol. Chem. 277:21749-21758.

5. Liu, S., D.A. Calderwood, and M.H. Ginsberg. 2000. Integrin cytoplasmic domain-binding proteins. J. Cell Sci. 113:3563-3571.

6. Franco, S.J., M.A. Rodgers, B.J. Perrin, J. Han, D.A. Bennin, D.R. Critchley, and A. Huttenlocher. 2004. Calpain-mediated proteolysis of talin regulates adhesion dynamics. Nat. Cell Biol. 6:977-983.

7. Shimonaka, M., K. Katagiri, T. Kakayama, N. Fujita, T. Tsuruo, O. Yoshie, and T. Kinashi. 2003. Rap1 translates chemokine signals to integrin activation, cell polarization, and motility across vascular endothelium under flow. J. Cell Biol. 161:417-427.

8. Katagiri, K., A. Maeda, M. Shimonaka, and T. Kinashi. 2003. RAPL, a novel Rap1-binding molecule, mediates Rap1-induced adhesion through spatial regulation of LFA-1. Nat. Immunol. 4:741-748.

9. Kim, M., C.V. Carman, and T.A. Springer. 2003. Bidirectional transmembrane signaling by cytoplasmic domain separation in integrins. Science. 301:1720-1725

10. Takagi, J., B.M. Petre, T. Walz, and T.A. Springer. 2002. Global conformational rearrangements in integrin extracellular domains in outsidein and inside-out signaling. Cell. 110:599-611.

11. Luo, B.-H., T.A. Springer, and J. Takagi. 2004. A specific interface between integrin transmembrane helices and affinity for ligand. PLoS Biol. 2:776-786.

12. Nishida, N., C. Xie, M. Shimaoka, Y. Cheng, T. Walz, and T.A. Springer. 2006. Activation of leukocyte beta2 integrins by conversion from bent to extended conformations. Immunity. 25:583-594.

13. Shimaoka, M., J. Takagi, and T.A. Springer. 2002. Conformational regulation of integrin structure and function. Annu. Rev. Biophys. Biomol. Struct. 31:485-516.

14. Yang, W., M. Shimaoka, A. Salas, J. Takagi, and T.A. Springer. 2004 Inter-subunit signal transmission in integrins by a receptor-like interaction with a pull spring. Proc. Natl. Acad. Sci. USA. 101:2906-2911.

15. Xiao, T., J. Takagi, J.-h. Wang, B.S. Coller, and T.A. Springer. 2004 Structural basis for allostery in integrins and binding of ligand-mimetic therapeutics to the platelet receptor for fibrinogen. Nature. 432:59-67.

16. Kim, M., C.V. Carman, W. Yang, A. Salas, and T.A. Springer. 2004 The primacy of affinity over clustering in regulation of adhesiveness of the integrin $\alpha_{\mathrm{L}} \beta_{2}$. J. Cell Biol. 167:1241-1253.

17. Dustin, M.L., and T.A. Springer. 1989. T cell receptor cross-linking transiently stimulates adhesiveness through LFA-1. Nature. 341:619-624.

18. Lollo, B.A., K.W.H. Chan, E.M. Hanson, V.T. Moy, and A.A. Brian. 1993. Direct evidence for two affinity states for lymphocyte function-associated antigen 1 on activated T cells. J. Biol. Chem. 268: 21693-21700

19. Bazzoni, G., and M.E. Hemler. 1998. Are changes in integrin affinity and conformation overemphasized? Trends Biochem. Sci. 23:30-34.

20. van Kooyk, Y., and C.G. Figdor. 2000. Avidity regulation of integrins: the driving force in leukocyte adhesion. Curr. Opin. Cell Biol. 12:542-547.

21. Stewart, M.P., A. McDowall, and N. Hogg. 1998. LFA-1-mediated adhesion is regulated by cytoskeletal restraint and by a $\mathrm{Ca}^{2+}$-dependent protease, calpain. J. Cell Biol. 140:699-707.

22. Krendel, M., and M.S. Mooseker. 2005. Myosins: tails (and heads) of functional diversity. Physiology (Bethesda). 20:239-251.

23. Berg, J.S., B.C. Powell, and R.E. Cheney. 2001. A millennial myosin census. Mol. Biol. Cell. 12:780-794.

24. Bresnick, A.R. 1999. Molecular mechanisms of nonmuscle myosin-II regulation. Curr. Opin. Cell Biol. 11:26-33.

25. Wessels, D., D.R. Soll, D. Knecht, W.F. Loomis, A. De Lozanne, and J. Spudich. 1988. Cell motility and chemotaxis in Dictyostelium amebae lacking myosin heavy chain. Dev. Biol. 128:164-177.

26. Maupin, P., C.L. Phillips, R.S. Adelstein, and T.D. Pollard. 1994. Differential localization of myosin-II isozymes in human cultured cells and blood cells. J. Cell Sci. 107:3077-3090.

27. Rochlin, M.W., K. Itoh, R.S. Adelstein, and P.C. Bridgman. 1995. Localization of myosin II A and B isoforms in cultured neurons. J. Cell Sci. 108:3661-3670. 
28. Straight, A.F., A. Cheung, J. Limouze, I. Chen, N.J. Westwood, J.R. Sellers, and T.J. Mitchison. 2003. Dissecting temporal and spatial control of cytokinesis with a myosin II Inhibitor. Science. 299:1743-1747.

29. Kovacs, M., J. Toth, C. Hetenyi, A. Malnasi-Csizmadia, and J.R. Sellers. 2004. Mechanism of blebbistatin inhibition of myosin II. J. Biol. Chem. 279:35557-35563.

30. Jana, S.S., S. Kawamoto, and R.S. Adelstein. 2006. A specific isoform of nonmuscle myosin II-C is required for cytokinesis in a tumor cell line. J. Biol. Chem. 281:24662-24670.

31. Campbell, J.J., J. Hedrick, A. Zlotnik, M.A. Siani, D.A. Thompson, and E.C. Butcher. 1998. Chemokines and the arrest of lymphoyctes rolling under flow conditions. Science. 279:381-384.

32. Shimaoka, M., M. Kim, E.H. Cohen, W. Yang, N. Astrof, D. Peer, A. Salas, A. Ferrand, and T.A. Springer. 2006. AL-57, a ligand-mimetic antibody to integrin LFA-1, reveals chemokine-induced affinity up-regulation in lymphocytes. Proc. Natl. Acad. Sci. USA. 103:13991-13996.

33. Sanchez-Madrid, F., and M.A. del Pozo. 1999. Leukocyte polarization in cell migration and immune interactions. EMBO J. 18:501-511.

34. Smith, A., Y.R. Carrasco, P. Stanley, N. Kieffer, F.D. Batista, and N Hogg. 2005. A talin-dependent LFA-1 focal zone is formed by rapidly migrating T lymphocytes. J. Cell Biol. 170:141-151.

35. Vicente-Manzanares, M., and F. Sánchez-Madrid. 2004. Role of the cytoskeleton during leukocyte responses. Nat. Rev. Immunol. 4:110-122.

36. Giannone, G., B.J. Dubin-Thaler, H.-G. Dobereiner, N. Kieffer, A.R. Bresnick, and M.P. Sheetz. 2004. Periodic lamellipodial contractions correlate with rearward actin waves. Cell. 116:431-443.

37. Cai, Y., N. Biais, G. Giannone, M. Tanase, G. Jiang, J.M. Hofman, C.H. Wiggins, P. Silberzan, A. Buguin, B. Ladoux, and M.P. Sheetz. 2006. Nonmuscle myosin IIA-dependent force inhibits cell spreading and drives F-actin flow. Biophys. J. 91:3907-3920.

38. Carman, C.V., and T.A. Springer. 2003. Integrin avidity regulation: are changes in affinity and conformation underemphasized? Curr. Opin. Cell Biol. 15:547-556.

39. Lu, C., M. Ferzly, J. Takagi, and T.A. Springer. 2001. Epitope mapping of antibodies to the $\mathrm{C}$-terminal region of the integrin $\beta 2$ subunit reveals regions that become exposed upon receptor activation. J. Immunol. 166:5629-5637.

40. Lu, C., M. Shimaoka, Q. Zang, J. Takagi, and T.A. Springer. 2001 Locking in alternate conformations of the integrin $\alpha \mathrm{L} \beta 2$ I domain with disulfide bonds reveals functional relationships among integrin domains. Proc. Natl. Acad. Sci. USA. 98:2393-2398.

41. Beglova, N., S.C. Blacklow, J. Takagi, and T.A. Springer. 2002. Cysteine-rich module structure reveals a fulcrum for integrin rearrangement upon activation. Nat. Struct. Biol. 9:282-287.

42. Smith, A., P. Stanley, K. Jones, L. Svensson, A. McDowall, and N Hogg. 2007. The role of the integrin LFA-1 in T-lymphocyte migration. Immunol. Rev. 218:135-146.
43. Bell, G.I. 1978. Models for the specific adhesion of cells to cells: a theoretical framework for adhesion mediated by reversible bonds between cell surface molecules. Science. 200:618-627.

44. Ridley, A.J., M.A. Schwartz, K. Burridge, R.A. Firtel, M.H. Ginsberg, G. Borisy, J.T. Parsons, and A.R. Horwitz. 2003. Cell migration: integrating signals from front to back. Science. 302:1704-1709.

45. Jacobelli, J., S.A. Chmura, D.B. Buxton, M.M. Davis, and M.F Krummel. 2004. A single class II myosin modulates T cell motility and stopping, but not synapse formation. Nat. Immunol. 5:531-538.

46. Zhang, H., J.S. Berg, Z. Li, Y. Wang, P. Lang, A.D. Sousa, A. Bhaskar, R.E. Cheney, and S. Stromblad. 2004. Myosin-X provides a motorbased link between integrins and the cytoskeleton. Nat. Cell Biol. 6:523-531.

47. Kupfer, A., P. Burn, and S.J. Singer. 1990. The PMA-induced specific association of LFA-1 and talin in intact cloned T helper cells. J. Mol. Cell. Immunol. 4:317-325.

48. Dustin, M.L., T.G. Bivona, and M.R. Philips. 2004. Membranes as messengers in T cell adhesion signaling. Nat. Immunol. 5:363-372.

49. Allingham, J.S., R. Smith, and I. Rayment. 2005. The structural basi of blebbistatin inhibition and specificity for myosin II. Nat. Struct. Mol. Biol. 12:378-379.

50. Jay, P.Y., and E.L. Elson. 1992. Surface particle transport mechanism independent of myosin II in Dictyostelium. Nature. 356:438-440.

51. Gupton, S.L., and C.M. Waterman-Storer. 2006. Spatiotemporal feedback between actomyosin and focal-adhesion systems optimizes rapid cell migration. Cell. 125:1361-1374.

52. Jeon, T.J., D.J. Lee, S. Merlot, G. Weeks, and R.A. Firtel. 2007. Rap1 controls cell adhesion and cell motility through the regulation of myosin II. J. Cell Biol. 176:1021-1033.

53. Smith, A., M. Bracke, B. Leitinger, J.C. Porter, and N. Hogg. 2003 LFA-1-induced T cell migration on ICAM-1 involves regulation of MLCK-mediated attachment and ROCK-dependent detachment. J. Cell Sci. 116:3123-3133.

54. Ginsberg, M.H., A. Partridge, and S.J. Shattil. 2005. Integrin regulation. Curr. Opin. Cell Biol. 17:509-516.

55. Chen, Z., O. Naveiras, A. Balduini, A. Mammoto, M.A. Conti, R.S Adelstein, D. Ingber, G.Q. Daley, and R.A. Shivdasani. 2007. The MayHegglin anomaly gene MYH9 is a negative regulator of platelet biogenesis modulated by the Rho-ROCK pathway. Blood. 110:171-179.

56. Constantin, G., M. Majeed, C. Giagulli, L. Piccib, J.Y. Kim, E.C Butcher, and C. Laudanna. 2000. Chemokines trigger immediate $\beta 2$ integrin affinity and mobility changes: differential regulation and roles in lymphocyte arrest under flow. Immunity. 13:759-769.

57. Giagulli, C., E. Scarpini, L. Ottoboni, S. Narumiya, E.C. Butcher, G. Constantin, and C. Laudanna. 2004. RhoA and $\zeta$ PKC control distinct modalities of LFA-1 activation by chemokines: critical role of LFA-1 affinity triggering in lymphocyte in vivo homing. Immunity. 20:25-35 